\title{
Variance of the likelihood ratio measure of bias
}

\author{
ETHEL MATIN and VINCENT VALLE \\ C. W. Post Center, Long Island University, Greenvale, New York
}

\begin{abstract}
The variance of the logarithm of the likelihood ratio, a commonly used measure of bias in signal detection theory, is derived for the case of an unbiased observer and two underlying normal distributions of equal variance. This variance increases with $d^{\prime}$ (the subject's sensitivity to the two stimuli involved in the discrimination). Accordingly, increased deviation of the In (LR) sample mean from a true mean of zero can be anticipated as signal level increases even when bias and sensitivity are essentially independent.
\end{abstract}

During the analysis of data obtained in studies of orientation sensitivity with yes-no signal detection methods, we noticed the following phenomenon: In absolute value, the experimentally obtained logarithm of the likelihood ratio $[\ln (\mathrm{LR})]$, a standard measure of bias, showed a strong tendency to increase as the physical difference between the two orientations involved in the discrimination increased. We were at first puzzled by this phenomenon, which is counterintuitive. Moreover, because sensitivity $\left(d^{\prime}\right)$ increases with increases in the physical difference between the two orientations, it seemed to imply a correlation between bias and sensitivity in our subjects' responding. Finally, there was no sign of such an increase when we measured bias with a distribution-free descriptive method, simply subtracting the proportion of false alarms from the proportion of misses.

We found no reference to the phenomenon described in the preceding paragraph in the signal detection literature and no published data that could be examined to see if other workers had obtained similar effects. However, the increase also occurred when we performed computer simulations of the behavior of an unbiased observer in short experimental runs (180 trials). This would be expected if the phenomenon is a general property of the paradigm rather than one peculiar to orientation discriminations or to our particular experimental setup. Further consideration showed that this is indeed the case: The experimentally obtained increase in $\ln (\mathrm{LR})$ with the magnitude of the signal reflects a theoretically expected increase in the variance of this measure and does not, per se, imply an increase in mean bias. Specifically, assuming an unbiased observer [i.e., mean $\ln (\mathrm{LR})=0$ ] and two underlying normal distributions of equal variance, we could expect the $\ln (\mathrm{LR})$ statistic to have a variance equal to $\left[\left(\mathrm{d}^{\prime}\right)^{2} \mathrm{PQ}\right] / \mathrm{NY}^{2}$,

This report was supported by Grant R01 EY02951 from the National Eye Institute and by an award from the Research Committee of the C. W. Post Center. Requests for reprints should be sent to: Ethel Matin, Department of Psychology, C. W. Post Center, Greenvale, NY 11548. where $d^{\prime}$ is the difference between the means of the two distributions in standard score units, $P$ and $Q$ are proportions of correct and incorrect responses, respectively, $\mathrm{N}$ is the size of the sample, and $\mathrm{Y}$ is the density of the standard normal deviate at the equal bias point.

Three factors contribute to the expected variance. The first two (combined as $\mathrm{PQ} / \mathrm{NY}^{2}$ ) result from the binomial variance of the proportions in the raw data and from the mapping of the proportions into $\mathrm{Z}$ scores. They are well-known in another context as the MüllerUrban weights (see Finney, 1971, for an explanation of the weights and for a discussion of their use in curvefitting procedures). The third factor, $\left(\mathrm{d}^{\prime}\right)^{2}$ can be derived for the yes-no paradigm and for Gaussian distributions of equal variance (the model described in Green $\&$ Swets, 1974 , p. $58-59$ ) by assuming a standardized decision variable, $\mathrm{X}$, with normal distributions under two hypotheses $\mathrm{H}_{0}$ and $\mathrm{H}_{1}\left[\mathrm{~N}(0,1)\right.$ and $\mathrm{N}\left(\mathrm{d}^{\prime}, 1\right)$, respectively], where $\mathrm{H}_{0}$ assumes the "noise alone" event and $\mathrm{H}_{1}$ assumes the "signal + noise" event of the classical model. Then,

$$
\begin{aligned}
L R= & f\left(x \mid H_{1}\right) / f\left(x \mid H_{0}\right) \\
= & \left(2 \pi \sigma^{2}\right)^{-1 / 2} e^{-\left(X-d^{\prime}\right)^{2} / 2} \\
& \left(2 \pi \sigma^{2}\right)^{-1 / 2} e^{-(X)^{2} / 2} \\
= & e^{\left[X d^{\prime}-\left(d^{\prime}\right)^{2}\right] / 2}
\end{aligned}
$$

and

$$
\ln (\mathrm{LR})=\mathrm{Xd}^{\prime}-\left[\left(\mathrm{d}^{\prime}\right)^{2} / 2\right],
$$

where $L R$ is the likelihood ratio and $\ln (\mathrm{LR})$ is its natural logarithm.

From Equation 4 above, it can be seen that the random variable $\ln (L R)$ is a linear function of the $\mathrm{N}(0,1)$ random variable $\mathrm{X}$, whose variance $\sigma^{2}(X)$ is 1 . Accordingly, the model predicts

$$
\sigma^{2}[\ln (\mathrm{LR})]=\left(\mathrm{d}^{\prime}\right)^{2} \sigma^{2}(\mathrm{X})=\left(\mathrm{d}^{\prime}\right)^{2}
$$


(expected variance without Müller-Urban weights). Because the data are in the form of proportions and must be mapped into standard normal scores before the model can be applied, the expected variance from the model $\left[\left(d^{\prime}\right)^{2}\right]$ should be combined with the weights. That is,

$$
\sigma^{2}[\ln (\mathrm{LR})]=\left[\left(\mathrm{d}^{\prime}\right)^{2} \mathrm{PQ}\right] / \mathrm{NY}^{2}
$$

(expected variance with Müller-Urban weights), where $\ln (\mathrm{LR})$ is the logarithm of the likelihood ratio, $\mathrm{d}^{\prime}$ is sensitivity, $\mathrm{P}$ and $\mathrm{Q}$ are proportions of correct and incorrect responses, respectively, $\mathrm{N}$ is the size of the sample, and $\mathrm{Y}$ is the density of the standard normal deviate at the equal bias point.

From Equation 6, it can be seen that larger sampling errors are expected for the $\ln (\mathrm{LR})$ as $\mathrm{d}^{\prime}$ increases. That is, even if the observer is essentially unbiased at all signal levels, we anticipate larger departures of the sample mean from the true mean of 0 that characterizes an unbiased observer (i.e., an observer who chooses $H_{1}$ if $X>d^{\prime} / 2$ and $H_{0}$ if $X<d^{\prime} / 2$ ). If this fact is not appreciated, the larger absolute values of the sample means can be misunderstood and interpreted as evidence for an increase in the subject's bias with an increase in sensitivity.

\section{REFERENCES}

Finney, D. (1971). Probit analysis. Cambridge: Cambridge University Press.

Green, D. M., \& Swets, J. (1974). Signal detection theory and psychophysics (rev. ed.). Huntington, NY: Krieger.

(Manuscript received for publication November 16, 1983.)

\section{NOTICES}

\section{Search for Editor of Memory \& Cognition}

The new editor is expected to begin handling submissions about January 1, 1985, or shortly thereafter. Nominations of qualified individuals should be sent to Robert G. Crowder, Department of Psychology, Yale University, Box 11A Yale Station, New Haven, CT 06520, by August 1, 1984.

\section{5th Annual Meeting of The Psychonomic Society, Inc.-Change of Dates for Meeting}

The dates for the 25th annual meeting of The Psychonomic Society, Inc., have been changed:

From-November 1-3, 1984

To-November 8-10, 1984

The meeting still will be held at the Hilton Palacio Del Rio, San Antonio, Texas. 